\title{
Effect of Lateral Walls on the Onset of Convective Motions
}

\author{
By K. Ukaji* and T. Matsuno \\ Department of Physics, Kyushu University, Fukuoka \\ (Manuscript received 2 October 1969, in revised form 19 March 1970)
}

\begin{abstract}
The critical Rayleigh number of a fluid layer confined in a box which is very thin in one horizontal direction and has lateral walls of finite thermal conductivity is studied theoretically. The lateral walls suppress the onset of convective motions not only mechanically but also thermally. The critical Rayleigh number, therefore, is far larger than that of a fluid layer, infinite in horizontal extent, 1708. The result coincides well with the experimental value obtained by Ukaji and Sawada (1969).
\end{abstract}

\section{Introduction}

In a paper by Ukaji and Sawada (1969), the relationship between the rate of vertical heat transport and the number of convection cells was investigated experimentally. The convection chamber used was a rectangular parallelepiped box with acrylic lateral walls and was very thin in one horizontal direction. The critical Rayleigh number obtained is $2.8 \times 10^{4}$, which is far larger than that of a fluid layer of infinite horizontal extent, 1708.

The problem of determining theoretically the condition for the onset of cellular convection was treated by many authors (see Chandrasekhar, 1961). Usually the model is a shallow horizontal fluid layer, infinite in horizontal extent and heated from below. It seems, however, these theories are not to be compared with experimental results using a box, because lateral confining walls may influence the onset of convective motions.

Elder (1967) showed that the critical Rayleigh number in a very thin (in one horizontal direction) box with "perfectly heat insulating" lateral walls is equivalent to that of a fluid in a porous medium, which had already been obtained by Lapwood (1948). The critical Rayleigh number $R_{c}$ in this case is

$$
R_{c}=4 \pi^{2} B,
$$

where $B$ is a non-dimensional quantity which expresses the frictional effect of lateral walls and

\footnotetext{
* Present affiliation: Dept. of Applied Physics, Fukuoka Univ., Fukuoka.
}

has a form $12(h / \varepsilon)^{2}$, where $h$ and $\varepsilon$ are the height and the thickness of the convection chamber respectively. Since the lateral friction suppresses convective motions, the critical Rayleigh number is larger than that of a fluid layer, infinite in horizontal extent. Application of this formula to the case of Ukaji and Sawada's exmeriments gives still too small value $\left(8.8 \times 10^{3}\right.$ vs. $2.8 \times 10^{4}$ ). Davis $(1967)$ investigated the critical Rayleigh number in a box with "perfectly heat conducting" lateral walls. According to the result, the lateral walls of infinite thermal conductivity suppress the onset of convective motions more strongly than the insulating lateral walls do. His numerical results do not cover the value of geometrical parameters applicable to the above mentioned exmeriment. But we can guess that his results will give more than $6 \times 10^{4}$.

Apparently their theories are inapplicable to experiments conducted by using a box with lateral walls of "finite thermal conductivity". In the present work, it is studied how the lateral walls of finite thermal conductivity influence the onset of convective motions. The result compares well with the experimental critical Rayeigh number obtained by Ukaji and Sawada (1969).

\section{Analysis}

Consider a thin rectangular parallelepiped fluid layer. Let $\varepsilon$ be the thickness, $h$ the height and $L$ the length. The thickness $\varepsilon$ is small compared to the height $h$, which in turn is very small compared to the length $L$. Let us use a Cartesian co-ordinate frame with 0 as its origin located at the center of the fluid layer, $x, y$ and $z$ being 
directed in the length, the thickness and vertically upward respectively. The temperatures of the top boundary surface, $z=\frac{1}{2} h$, and of the bottom surface, $z=-\frac{1}{2} h$, are kept constant at $T_{c}$ and $T_{w}$ respectively $\left(\Delta T \equiv T_{w}-T_{c}>0\right)$. Since $L \gg h$ $(>\varepsilon)$, we may consider that the fluid layer is unbounded horizontally in one direction (say in the $x$-direction).

a) The equations of motion and heat conduction for fluid.

Making the so-called Boussinesq approximation, the equations of motion, the equation of continuity and the equation of heat conduction are written as follows:

$$
\begin{aligned}
& \frac{\partial u}{\partial t}=-\frac{\partial}{\partial x}\left(-\frac{p}{\rho}\right)+\nu \nabla^{2} u, \\
& \frac{\partial v}{\partial t}=-\frac{\partial}{\partial y}\left(\frac{p}{\rho}\right)+\nu \nabla^{2} v, \\
& \frac{\partial w}{\partial t}=-\frac{\partial}{\partial z}\left(\frac{p}{\rho}\right)+\nu \nabla^{2} w+g \alpha \theta, \\
& \frac{\partial u}{\partial x}+\frac{\partial v}{\partial y}+-\frac{\partial w}{\partial z}=0, \\
& \frac{\partial \theta}{\partial t}=\beta w+\kappa \nabla^{2} \theta .
\end{aligned}
$$

The notations used above are

$$
\nabla^{2}:=\frac{\partial^{2}}{\partial x^{2}}+\frac{\partial^{2}}{\partial y^{2}}+\frac{\partial^{2}}{\partial z^{2}},
$$

$u, v, w$ : velocity components in the $x-, y$ - and $z$-directions respectively,

$\beta$ : undisturbed vertical temperature gradient, $\frac{\Delta T}{h}$,

$p$ : pressure perturbation due to convective motions,

$\theta$ : temperature perturbation due to convective motions,

$\kappa$ : thermometric conductivity of the fluid,

$\rho$ : undisturbed density (constant),

$g$ : acceleration of gravity,

$\alpha$ : coefficient of thermal expansion,

$\nu$ : kinematic viscosity.

Davis (1967) showed that the prefered convective mode in a box is rolls with axes parallel to the shorter side. Then, taking $y$ as shorter side, fluid motions are confined to the $(x, z)$-plane and we may put $v \equiv 0^{\dagger}$.

$\dagger$ These features are confirmed by experiments (Elder, 1967; Ukaji and Sawada, 1969).
Then eliminating $u$ and $p / \rho$ from eqs. (1), (3) and (4), we get

$$
\begin{gathered}
\frac{\partial}{\partial t}\left[\begin{array}{c}
\left.\frac{\partial^{2}}{\partial x^{2}}+\frac{\partial^{2}}{\partial z^{2}}\right] \\
\left.+\frac{\partial^{2}}{\partial z^{2}}\right]
\end{array}\right] \nabla^{2} w . \\
\end{gathered}
$$

Since $\varepsilon<h$, lateral walls at $y= \pm \varepsilon / 2$ may suppress the fluid motion more than the top and the bottom boundaries $\left(z= \pm \frac{1}{2} h\right)$ do. Therefore, the nature of the top and the bottom surfaces, being either rigid or free, may not affect the magnitude of the critical Rayleigh number so much, provided that the rigid boundary conditions are taken for lateral walls. So we adopt the free boundary conditions at the top and the bottom surfaces, for simplification, i.e.

$$
w=\frac{\partial^{2} w}{\partial z^{2}}=0 \quad \text { for } z= \pm \frac{1}{2} h .
$$

Assuming that the top and the bottom boundary surfaces are perfectly heat conducting, the thermal boundary conditions are

$$
\theta=0 \quad \text { for } z= \pm \frac{1}{2} h .
$$

According to Rayleigh (1916), the ground mode solutions for $w$ and $\theta$ in this case are written as

$$
\begin{aligned}
& w=W(y) \cdot \cos \frac{\pi}{h} z \cdot e^{i k x+q t}, \\
& \theta=\Theta(y) \cdot \cos \frac{\pi}{h} z \cdot e^{i k x+q t},
\end{aligned}
$$

where $k$ and $q$ are the wave number in the direction of the longer horizontal dimension and the growth rate of the disturbance, respectively. $w$ and $\theta$ given as above satisfy eqs. (5) and (6) and boundary conditions (7) and (8), provided that $W$ and $\Theta$ are solutions to the equations,

$$
\begin{array}{r}
q \Theta=\beta W+\kappa\left[\frac{d^{2}}{d y^{2}}-\frac{\pi^{2}}{h^{2}}-k^{2}\right] \Theta, \\
q\left[-\frac{\pi^{2}}{h^{2}}+k^{2}\right] W=g \alpha k^{2} \Theta+\nu\left[\begin{array}{c}
\pi^{2} \\
h^{2}
\end{array}\right. \\
\left.\quad+k^{2}\right]\left[\begin{array}{c}
d^{2} \\
\left.-d y^{2}-\frac{\pi^{2}}{h^{2}}-k^{2}\right] W .
\end{array}\right.
\end{array}
$$

Since we are interested in determination of the critical Rayleigh number, hereafter we shall consider only a marginal state, i.e. $q=0$ in eqs. (11) and (12).

b) The equation of heat conduction for the 
walls.

As we consider a marginal state, the equation of heat conduction for the lateral confining walls is

$$
\nabla^{2} \theta^{\prime}=0
$$

where $\theta^{\prime}$ is the perturbed part of temperature of the wall, caused by convective motions of the fluid. Since there should be no temperature difference between the wall and the interior fluid on their contact surface, $\theta^{\prime}$ has the same functional form as $\theta$ in the $x$ - and $z$-directions. Therefore we put

$$
\theta^{\prime}=\Theta^{\prime}(y) \cdot \cos \frac{\pi}{h} z \cdot e^{i k x} .
$$

Then (13) turns to

$$
\left(\frac{d^{2}}{d y^{2}}--\frac{\pi^{2}}{h^{2}}-k^{2}\right) \Theta^{\prime}=0 .
$$

c) Boundary conditions.

Let us assume there is no heat leakage from the walls to the ambient air. Then the thermal boundary conditions at the outside boundaries of the walls are

$$
\frac{d \Theta}{d y}=0 \quad \text { for } y= \pm\left(\frac{\varepsilon}{2}+\delta\right)
$$

where $\delta$ is the thickness of the wall. At the interfaces between the wall and the fluid, heat flow should be continuous in addition to the continuity of temperature;

$$
\begin{aligned}
& \Theta=\Theta^{\prime}, \\
& \lambda \frac{d \Theta}{d y}=\lambda^{\prime} \frac{d \Theta^{\prime}}{d y} \quad \text { for } y= \pm \frac{\varepsilon}{2} .
\end{aligned}
$$

Here $\lambda$ and $\lambda^{\prime}$ are the thermal conductivities of the fluid and the wall respectively. As the condition for fluid motions, we shall assume that there is no motion at the walls, i.e. a rigid boundary condition;

$$
W=0 \quad \text { for } y= \pm \frac{\varepsilon}{2} \text {. }
$$

The equations (11), (12) and (15), together with the boundary conditions (16) through (19), form a complete set to determine $W, \Theta$ and $\Theta^{\prime}$. In the subsequent part of this section we shall obtain approximate solution to this system. Note that the total order of differentiation of the equations for fluid motion and temperature perturbation has been reduced to four by the assumption $v \equiv 0$. Then a boundary condition at lateral walls $v=0$ is automatically satisfied and another condition $u=0$ is degenerated to the condition $w=0$.

\section{Solution to wall temperature equation.}

The solution to (15) and (16) is readily obtained as

$$
\Theta^{\prime}=\frac{\Theta_{b}}{\cosh n \delta} \times\left\{\begin{array}{c}
\cosh n\left(y-\frac{\varepsilon}{2}-\delta\right) \\
\text { for } \frac{\varepsilon}{2} \leqq y \leqq \frac{\varepsilon}{2}+\delta \\
\cosh n\left(y+\frac{\varepsilon}{2}+\delta\right) \\
\text { for }-\frac{\varepsilon}{2}-\delta \leqq y \leqq-\frac{\varepsilon}{2},
\end{array}\right.
$$

where $\Theta_{b}$ is the temperature of the boundary surfaces and

$$
n=\sqrt{-\frac{\pi^{2}}{h^{2}}+k^{2}}
$$

Approximate solutions for fluid motion and temperature.

Next we shall seek for solution to (11) and (12). Eliminating $W$ between (11) and (12), we have

$$
\left(\frac{d^{2}}{d y^{2}}-n^{2}\right)^{2} \Theta-\frac{g \alpha \beta}{\kappa \nu} \cdot \frac{k^{2}}{n^{2}} \circledast=0 .
$$

General solutions of the above equation (with even symmetry with $y=0$ ) are obtained as

$$
\Theta=C_{1} \cosh \alpha_{1} y+C_{2} \cosh \alpha_{2} y,
$$

where $\alpha_{1}$ and $\alpha_{2}$ are roots of the equations,

$$
\begin{aligned}
\alpha_{1}{ }^{2} & =n^{2}+\sqrt{\frac{g \alpha \beta}{\kappa \nu} \cdot \frac{k^{2}}{n^{2}}} \\
\text { and } \alpha_{2}{ }^{2} & =n^{2}-\sqrt{\frac{g \alpha \beta}{\kappa \nu} \cdot \frac{k^{2}}{n^{2}}}
\end{aligned}
$$

If we apply the boundary conditions to the solution (22), we could get a characteristic equation to determine the critical Rayleigh number. However, as seen from (22) and (23) it must be a transcendental equation and may be difficult to deal with. So we shall adopt an approximate method to get solutions to (11) and (12), taking account of a special configuration of our convection chamber. The procedure described in the following might be equivalent with approximating (22) by a power series, though it is not done explicitly.

At first we shall obtain equations for integrated 
quantities. It follows from (11) and (12),

$$
\begin{gathered}
0=\beta \bar{W}-\kappa\left[\frac{\pi^{2}}{h^{2}}+k^{2}\right] \bar{\Theta}+\frac{\kappa}{\varepsilon}\left|\frac{d \Theta}{d y}\right|_{-\varepsilon / 2}^{+\varepsilon / 2}, \\
\begin{array}{c}
0=g \alpha k^{2} \bar{\Theta}-\nu\left[\frac{\pi^{2}}{h^{2}}+k^{2}\right]^{2} \bar{W} \\
+\frac{\nu}{\varepsilon}\left[-\frac{\pi^{2}}{h^{2}}+k^{2}\right]\left|\frac{d W}{d y}\right|_{-\varepsilon / 2}^{+\varepsilon / 2},
\end{array}
\end{gathered}
$$

where

$$
\begin{aligned}
& \bar{\Theta}=\frac{1}{\varepsilon} \int_{-\varepsilon i 2}^{+\varepsilon / 2} \Theta d y, \\
& \bar{W}=\frac{1}{\varepsilon} \int_{-\varepsilon / 2}^{+\varepsilon / 2} W d y .
\end{aligned}
$$

The last term of (24) expresses heat leakage from the fluid to the walls and the last term of (25) expresses the frictional effect of the walls, while the second term of each equation arises from diffusion effect of heat and momentum within the fluid.

Next we shall obtain relationship between

$$
\left|\frac{d W}{d y}\right|_{-\varepsilon / 2}^{+\varepsilon / 2}\left(\left|\frac{d \Theta}{d y}\right|_{-\varepsilon / 2}^{+\varepsilon / 2}\right) \text { and } \bar{W}(\vec{\Theta}) \text {. }
$$

As we are dealing with a fluid motion between walls of very small separation, we may assume that it is locally a plane Poiseulle flow, i.e.

$$
W=W_{0}\left(1-\frac{4}{\varepsilon^{2}} y^{2}\right),
$$

where $W_{0}$ is the maximum $W$. The boundary condition (19) is satisfied by this assumption.

It follows from (28)

$$
|d W|_{-\varepsilon / 2}^{+\varepsilon / 2}=-\frac{8}{\varepsilon} W_{0}=-\frac{12}{\varepsilon} \bar{W} .
$$

Corresponding to (28), we shall assume the func$t^{\text {ional form of } \Theta \text { as }}$

$$
\Theta=\left(\Theta_{0}-\Theta_{b}\right)\left(1-\frac{4}{\varepsilon^{2}} y^{2}\right)+\Theta_{b},
$$

where $\Theta_{0}$ is the maximum $\Theta$ and $\Theta_{b}$ is the tempeture at the boundary surface. The condition (17) is fulfilled. From the definition of $\bar{\Theta}(26)$, we have

$$
\bar{\Theta}=\frac{1}{3}\left(2 \Theta_{0}+\Theta_{b}\right) .
$$

It follows from (18), (20) and (30) that

$$
\left(\Theta_{0}-\Theta_{b}\right) \frac{4}{\varepsilon}=\gamma n \Theta_{b} \tanh n \delta,
$$

where $\gamma=\frac{\lambda^{\prime}}{\lambda}$.

Eliminating $\Theta_{0}$ between (31) and (32), we get

$$
\bar{\Theta}=\Theta_{b}\left[1+\frac{\varepsilon}{6} \gamma n \tanh n \delta\right] .
$$

Then $\left|\frac{d \Theta}{d y}\right|_{-\varepsilon / 2}^{+\varepsilon / 2}$ may be expressed in terms of $\Theta$ as

$$
\begin{aligned}
\left|\frac{d \Theta}{d y}\right|_{-\varepsilon / 2}^{+\varepsilon / 2} & =\gamma\left|\frac{d \Theta^{\prime}}{d y}\right|_{-\varepsilon / 2}^{+\varepsilon / 2} \\
& =-2 \gamma n \Theta_{b} \tanh n \delta \\
& =-\frac{2 \gamma n \tanh n \delta}{1+\frac{\varepsilon}{6} \gamma n \tanh n \delta} \bar{\Theta} .
\end{aligned}
$$

Inserting (29) and (34) into eqs. (25) and (24) respectively, we get

$$
\begin{aligned}
& \frac{g \alpha k^{2}}{\nu} \bar{\Theta}=\left[\frac{\pi^{2}}{h^{2}}+k^{2}\right]\left[\frac{\pi^{2}}{h^{2}}+k^{2}+\frac{12}{\varepsilon^{2}}\right] \bar{W}, \\
& \frac{\beta}{\kappa} \bar{W}=\left[\frac{\pi^{2}}{h^{2}}+k^{2}+\frac{2 \gamma n \tanh n \delta}{\varepsilon\left(1+\frac{\varepsilon}{6} \gamma n \tanh n \delta\right)}\right] \bar{\Theta} .
\end{aligned}
$$

Eqs. (35) and (36) may be made dimensionless. For this purpose, let us introduce following quantities;

$$
\begin{aligned}
\varepsilon_{0} & =-\frac{\varepsilon}{h}, \quad \delta_{0}=\frac{\delta}{h}, \\
a^{2} & =k^{2} h^{2}, \quad K_{1}=\frac{12}{\varepsilon_{0}^{2}}, \\
K_{2} & =\frac{2 \gamma \sqrt{\pi^{2}+a^{2}} \tanh \delta_{0} \sqrt{\pi^{2}+a^{2}}}{\varepsilon_{0}\left[1+\frac{\varepsilon_{0}}{6} \gamma \sqrt{\pi^{2}+a^{2}} \tanh \delta_{0} \sqrt{\pi^{2}+a^{2}}\right]} .
\end{aligned}
$$

Then eqs. (35) and (36) may be rewritten as follows:

$$
\begin{aligned}
& \frac{g \alpha h^{2}}{\nu} a^{2} \bar{\Theta}=\left(\pi^{2}+a^{2}\right)\left(\pi^{2}+a^{2}+K_{1}\right) \bar{W}, \\
& \frac{\beta h^{2}}{\kappa} \bar{W}=\left(\pi^{2}+a^{2}+K_{2}\right) \bar{\Theta} .
\end{aligned}
$$

If $\bar{\Theta}$ and $\bar{W}$ are not identically zero, we have

$$
R=\frac{\left(\pi^{2}+a^{2}\right)\left(\pi^{2}+a^{2}+K_{1}\right)\left(\pi^{2}+a^{2}+K_{2}\right)}{a^{2}},
$$

where $R\left(\equiv \frac{g \alpha \beta h^{4}}{\kappa \nu}\right)$ is the Rayleigh number. The critical Rayleigh number is the smallest value of $R$ in the marginal state. From (40), it is immediately seen that $R_{c}$ is a function of both $K_{1}$ and $K_{2} . \quad K_{1}$ contains $\varepsilon_{0}$ the ratio of the thickness $(\varepsilon)$ to the height $(h)$ of the convection chamber and expresses the frictional effect of the lateral walls. On the other hand, $K_{2}$ contains $\gamma$ the ratio of the thermal conductivity of the lateral wall $\left(\lambda^{\prime}\right)$ to that of the fluid $(\lambda)$ and expresses the 
effect of the heat exchange between the fluid and the walls. It implies that the onset of convection is affected both mechanically and thermally by the lateral walls.

\section{Results and discussions}

We can get from (40) the critical Rayleigh numbers for special cases.

a) A convection chamber is large in both horizontal directions $\left(\varepsilon_{0}=\infty\right)$.

It follows from (40) that

$$
R=\frac{\left(\pi^{2}+a^{2}\right)^{3}}{a^{2}}
$$

therefore

$$
\begin{aligned}
R_{c} & \equiv \frac{g \alpha \Delta T_{c r}}{\kappa \nu} h^{3} \\
& =\frac{27}{4} \pi^{4} \quad \text { for } a=\frac{\pi}{\sqrt{2}},
\end{aligned}
$$

where $\Delta T_{c r}$ is the critical temperature difference. This solution (41) has been obtained by Rayleigh (1916).

b) A convection chamber is very thin $\left(\varepsilon_{0} \ll 1\right.$, $\left.\pi^{2}+a^{2} \ll K_{1}\right)$. The lateral walls are thinner $\left(\delta_{0} \ll \varepsilon\right)$ and their thermal conductivity is not so large $(\gamma \lesssim 1)$. Then $K_{2} \ll \pi^{2}+a^{2 \dagger}$.

We obtain from (40)

$$
R=\frac{\left(\pi^{2}+a^{2}\right)^{2}}{a^{2}} \cdot \frac{12}{\varepsilon_{0}^{2}},
$$

therefore

$$
\begin{aligned}
R_{c}{ }^{*} & \equiv \frac{g_{\alpha} \Delta T_{c r}}{\kappa \nu} \cdot \frac{\varepsilon^{2}}{12} h \\
& =4 \pi^{2} \quad \text { for } a=\pi .
\end{aligned}
$$

This is the case treated by Elder (1967).

c) A convection chamber is very thin $\left(\varepsilon_{0} \ll 1\right.$, $\pi^{2}+a^{2} \ll K_{1}$ ), the lateral walls are very thick $\left(\delta_{0} \gg 1\right)$ and their thermal onductivity is finite $(\gamma \sim 1)$.

It follows from (40)

$$
R=\frac{\left(\pi^{2}+a^{2}\right)^{3 / 2}}{a^{2}} \cdot \frac{24 \gamma}{\varepsilon_{0}{ }^{3}},
$$

accordingly

$\dagger$ It the lateral walls are of finite thickness and their thermal conductivity is extremely small $\left(\gamma \ll \varepsilon_{0}\right)$, we have the same equation. In the actual experiments, however, these conditions are unlikely to hold.

$$
\begin{aligned}
R_{c} * * & \equiv \frac{g \alpha \Delta T_{c r}}{\kappa \nu} \cdot \frac{\varepsilon^{3}}{24 \gamma} \\
& =\frac{3^{3 / 2}}{2} \pi \quad \text { for } a=\sqrt{2} \pi .
\end{aligned}
$$

$R_{c}{ }^{*}$ and $R_{c}{ }^{* *}$ are the re-defined critical Rayleigh numbers.

d) The thermal conductivity of the lateral walls is infinite $(\gamma=\infty)$.

We get from (40)

$$
R=\frac{\left(\pi^{2}+a^{2}\right)\left(\pi^{2}+a^{2}+K_{1}\right)^{2}}{a^{2}} .
$$

Davis (1967 Figure 5) treated this case for $0.25 \leqq \varepsilon_{0} \leqq 6$. According to his paper, for $\varepsilon_{0}=0.25$,

$$
R_{c} \fallingdotseq 6 \times 10^{4} \text { and } a \fallingdotseq 5.1 \text {. }
$$

On the other hand, (44) gives for $\varepsilon_{0}=0.25$ and $K_{1}=192$

$$
R_{c}=7.1 \times 10^{4} \quad \text { for } a=5.4 \text {. }
$$

This critical Rayleigh number is about $15 \%$ larger than that obtained by Davis. It seems that this difference is due to assuming the approximate solutions to $W$ and $\Theta$ in our analysis.

The convection chamber used in the experiment reported by Ukaji and Sawada (1969) is a thin rectangular parallelepiped box whose dimensions are $284 \mathrm{~mm}$ long, $42 \mathrm{~mm}$ high and $9.8 \mathrm{~mm}$ thick. The lateral walls $20 \mathrm{~mm}$ thick acrylic resin, the thermal conductivity of which is $5 \times 10^{-4} \mathrm{cal} \cdot \mathrm{cm}^{-1}$. $\sec ^{-1} \cdot{ }^{\circ} \mathrm{C}^{-1}$. The fluid is silicone oil whose thermal conductivity is $3.8 \times 10^{-4} \mathrm{cal} \cdot \mathrm{cm}^{-1} \cdot \mathrm{sec}^{-1}$. ${ }^{\circ} \mathrm{C}^{-1}$. From these we have $\varepsilon_{0}=0.23, \delta_{0}=0.48$, $\gamma=1.3, K_{1}=220$ and $K_{2}=43$. Then we obtain the critical Rayleigh number as

$$
R_{c}=2.8 \times 10^{4} \text { for } a=3.7 \text { (see Fig. 1). }
$$

This value of $R_{c}$ is in good agreement with the experimental value, $R_{c}=2.8 \times 10^{4}$ (see Fig. 2 ). The half wave length of observed marginally unstable roll was $3.6 \mathrm{~cm}$ or $a=3.7$.

The several critical Rayleigh numbers which correspond to different thermal conductivity of lateral walls are summarized in the Table 1 . The results show that the lateral walls tend to suppress the onset of convective motions. The mechanical effect is more or less apparent. On the other hand, the thermal effect, which is often overlooked, also causes a great change of the critical Rayleigh number for the convection in a box with thick lateral walls. Heat flow through the wall will reduce the temperature perturbation 
in the fluid and suppresses the development of convective motions.

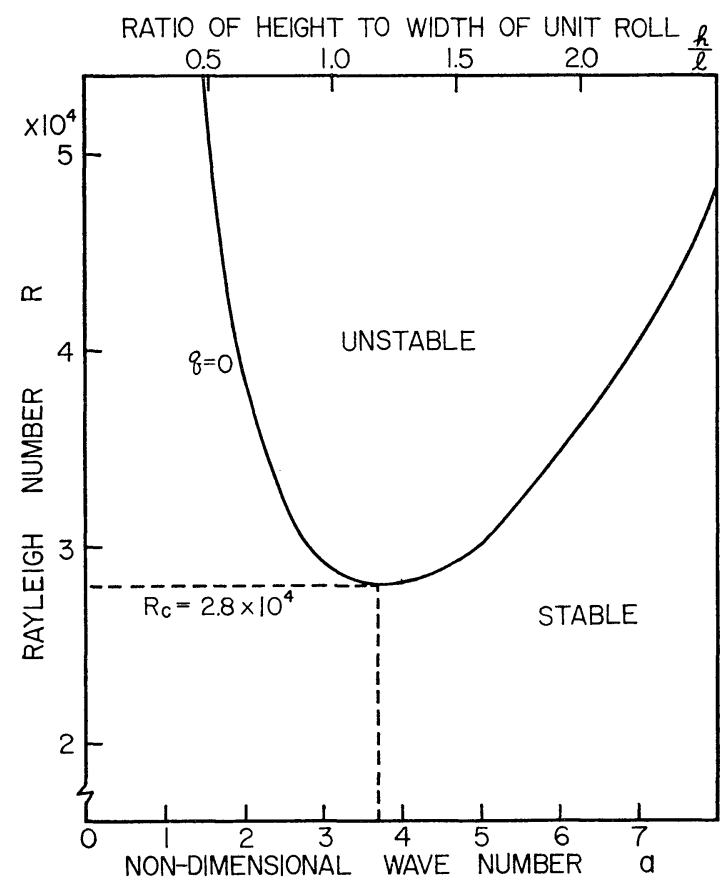

Fig. 1. The Rayleigh number at which convective motions develop as a function of non-dimensional wave number, $a$. Instead of $a$, the ratio of the height ( $h$ ) to the width $(l)$ of a convection roll, $h / l$, may be used. The critical Rayleigh number, $R_{c}=2.8 \times 10^{4}$ corresponds to $a=3.7$, also $h / l=1.2$.

Table 1. The critical Rayleigh numbers corresponding to different physical situations of lateral walls.

\begin{tabular}{c|c|c|c}
\hline$\varepsilon_{0}$ & $\gamma$ & $R_{c}$ & $a$ \\
\hline$\infty$ & finite & $6.58 \times 10^{2}$ & 2.22 \\
0.23 & 0 & $8.8 \times 10^{3}$ & 3.14 \\
0.23 & $1.3\left(\delta_{0}=0.48\right)$ & $2.8 \times 10^{4}$ & 3.7 \\
0.23 & $\infty$ & $9.0 \times 10^{4}$ & 5.6 \\
\hline
\end{tabular}

\section{Acknowledgements}

The authors wish to express their heartfelt thanks to Prof. R. Sawada, Kyushu University, for his valuable discussions and to Miss Y. Satō for her drawing figures.

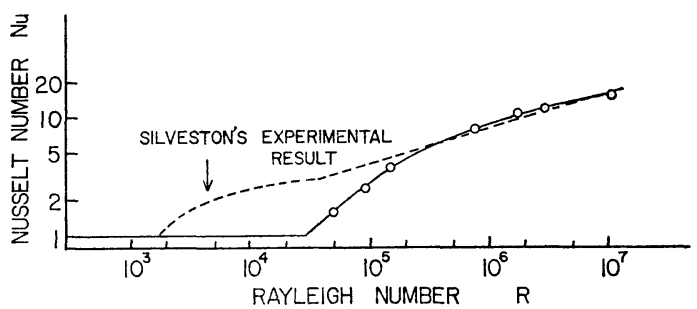

Fig. 2. Relationship between the rate of vertical heat transport, $N_{u}$ and the Rayleigh number, $R$. [Taken from Fig. 4 of Ukaji and Sawada (1969).] The convection chamber used is a thin rectangular parallelepiped box. The lateral walls are of finite thermal conductivity. Circles show results of the experiment. A solid curve is drawn simly to connect them and extended rather subjectively to meet around $R=R_{c}$ $\doteqdot 2.8 \times 10^{4}$ with a horizontal line, $N_{u}=1$, for non-convection regime. The broken curve shows Silveston's experimental result (1958). His convection chamber is a circular shallow vase and the critical Rayleigh number, $1700 \pm 51$, is in good agreement with the theoretical one of a fluid layer, infinite in horizontal extent, 1708.

\section{References}

Chandrasekhar, S., 1961: Hydrodynamic and hydromagnetic stability. Oxford University Press, $652 \mathrm{pp}$.

Davis, S.H., 1967: Convection in a box: linear theory. J. Fluid Mech., 30, 465-478.

Elder, J.W., 1967: Steady free convection in a porous medium heated from below. J. Fluid Mech., 27, 29-48.

Lapwood, E.R., 1948: Convection of a fluid in a porous medium. Proc. Camb. Phil. Soc., 44, 508521.

Rayleigh, Lord, 1916: On convection currents in a horizontal layer of fluid when the higher temperature is on the under side. Phil. Mag., 32, 529-546.

Silveston, P.L., 1958: Wärmedurchgang in waagerechten Flüssigkeitsschichten. Forsch. Ing. Wes., 24, 29-32 and 59-69.

Ukaji, K. and R. Sawada, 1969: The convective heat transport and the number of convection cells. J. Meteor. Soc. Japan, 47, 451-456. 


\title{
対流槽の側壁が対流の発生に及ぼす効果
}

\author{
宇加治 一 雄*. 松 野 太 郎 \\ 九州大学, 理学部
}

有限の熱伝導率をもつ側壁にかこまれ，一水平方向に薄い流体層の臨界レーリー数の計算を行った。

このような対流槽においては, 流体と側壁との間で行なわれる熱のやりとりと, 流体が側壁かららける摩擦のため に，対流の発生が抑制される，それゆ元，この場合の臨界レーリ一数は，水平方向に無限に広がった流体層の臨界レ

ーリー数, 1708，よりも非常に大きくなる. 計算結果は実験值 (Ukaji and Sawada, 1969) とよく一致した。

* 現在の所属 : 福岡大学, 理学部 